\title{
DESENVOLVIMENTO DE MÉTODO ANALÍTICO POR CLAE EM COMPRIMIDOS DE BENZNIDAZOL PARA A DOENÇA DE CHAGAS
}

Ana Luiza Maurer da Silva, José Lamartine Soares Sobrinho e Pedro José Rolim Neto*

Departamento de Ciências Farmacêuticas, Universidade Federal de Pernambuco, Av. Prof. Arthur de Sá, s/n, 50740-521

Recife - PE, Brasil

Rosali Maria Ferreira da Silva, Flávia Patrícia Morais de Medeiros e Leduar Guedes de Lima

Laboratório Farmacêutico do Estado de Pernambuco, Largo de Dois Irmãos, 1117, 52171-010 Recife - PE, Brasil

Recebido em 28/6/06; aceito em 11/12/06; publicado na web em 17/7/07

\begin{abstract}
DEVELOPMENT OF AN HPLC ANALYTICAL METHOD FOR BENZNIDAZOL TABLETS FOR THE TREATMENT OF CHAGAS DISEASE. The analytical method of high performance liquid chromatography (HPLC) for the assay of benznidazole in tablets was developed and validated following the requirements of regulatory agencies. The method used as mobile phase acetonitrile:wather 1:1, a C18 column of $12.5 \mathrm{~cm}$ length $\mathrm{x} 4 \mathrm{~mm}$ id, $5 \mu \mathrm{m}$ particles and $\lambda=316 \mathrm{~nm}$. The statistical analysis of the results demonstrated that the method satisfies all parameters so as to be considered a safe and efficient analytical alternative of low cost for laboratory routine.
\end{abstract}

Keywords: development of method; validation; high performance liquid chromatography.

\section{INTRODUÇÃO}

A doença de Chagas é uma enfermidade endêmica na América Latina, afetando em torno de 13 milhões de pessoas ${ }^{1}$. Seu agente etiológico é o Trypanosoma cruzi, um protozoário hemoflagelado da família Trypanosomatidae e ordem Kinetoplastida ${ }^{2}$. A incidência anual estimada de novos casos da doença decaiu de 700.000 800.000 na década de 80 para aproximadamente 200.000 novos casos $^{1}$.

As doenças negligenciadas tais como leishmaniose, tripanossomíase, doença de Chagas e malária têm impacto devastador sobre a humanidade ${ }^{3}$. O benznidazol (N-benzil-2-nitro-1-imidazolacetamida) é um antiparasitário, derivado nitroimidazol, com ação tripanossomicida, que vem sendo utilizado, desde 1973, no tratamento etiológico da doença de Chagas ${ }^{4}$.

Alguns métodos analíticos para quantificação do benznidazol têm sido divulgados ao longo do tempo, utilizando diversas técnicas como eletroquímica ${ }^{5-7}$, polarografia ${ }^{8}$, espectrofotometria $U^{9}$ e cromatografia líquida de alta eficiência ${ }^{10}$, porém na sua grande maioria essas quantificações do ativo ocorrem em fluidos biológi$\cos ^{5-7,10}$. Para a fabricação de comprimidos à base de benznidazol, visto a falta de métodos nos compêndios oficiais, faz-se necessário o desenvolvimento e a validação de um método que atenda às especificações dos órgãos reguladores ${ }^{11-13}$.

Para garantir que um novo método analítico gere informações confiáveis e interpretáveis sobre a amostra, ele deve sofrer uma avaliação denominada validação. A validação inclui especificação dos requisitos do método; determinação das características do método; verificação de que os requisitos podem ser atendidos com o uso do método e uma declaração sobre a validade do método ${ }^{14-19}$.

O presente trabalho tem como objetivo o desenvolvimento e validação de método analítico para doseamento de comprimidos à base de benznidazol, de acordo com a RE 899 da Agência Nacional de Vigilância Sanitária (ANVISA) ${ }^{13}$.

*e-mail: prolim@globo.com

\section{PARTE EXPERIMENTAL}

\section{Materiais e métodos}

Matéria-prima, reagentes e vidrarias

Para as etapas que corresponderam ao desenvolvimento do método e sua validação, foram utilizados padrão de trabalho e matéria-prima (Lote LAFEPE 13871) fornecidos pela Roche ${ }^{\circledR}$, amido de milho, lactose malha 200, estearato de magnésio, talco e os comprimidos de benznidazol fabricados no LAFEPE $^{\circledR}$ (Lote 05030569). Os reagentes utilizados no estudo foram: acetonitrila grau HPLC Tedia ${ }^{\circledR}$, metanol grau HPLC Tedia ${ }^{\circledR}$ e água ultrapurificada obtida por Milli-Q Millipore ${ }^{\circledR}$. As colunas testadas tiveram empacotamento $\mathrm{C} 8$ e $\mathrm{C} 18$, apresentando as seguintes especificações: C8 (Symmetry $^{\circledR}, 25 \mathrm{~cm} \mathrm{x} \mathrm{4,6} \mathrm{mm} \mathrm{di;} \mathrm{partícula} 5$ $\mu \mathrm{m}), \mathrm{C} 18\left(\mu\right.$ Bondpack $^{\circledR}, 300$ x 3,9 mm di; partícula $10 \mu \mathrm{m}$ e Lichrospher ${ }^{\circledR} 12,5 \mathrm{~cm} \times 4,0 \mathrm{~mm}$ di). A vidraria utilizada foi certificada por lote, sendo da marca Pirex ${ }^{\circledR}$.

\section{Desenvolvimento do método analítico}

Primeiramente foi realizada uma varredura em espectrofotômetro Shimadzu ${ }^{\circledR}$ com duplo feixe, modelo N-2401 PC, com detector de 190 a $800 \mathrm{~nm}$, possuindo lâmpadas de deutério e tungstênio. Sua calibração foi realizada através de padrões de referência, rastreados pelo NIST ("National Institute of Standards and Technology"). A amostra de comprimidos testada foi diluída em metanol Merck ${ }^{\circledR}$ até uma concentração final de $40 \mathrm{mg} \mathrm{L}^{-1}$, sendo a varredura programada em uma faixa entre 200 a $800 \mathrm{~nm}$, objetivando em confirmar o comprimento de absorvância máximo citado pela literatura.

Visando redução de custo e de tempo de análise, realizou-se estudo em Cromatografia Líquida de Alta Eficiência (CLAE) das amostras de comprimidos preparadas a $100 \mathrm{mg} \mathrm{L}^{-1}$, utilizando quatro diluições em diferentes misturas de solventes (metanol, acetonitrila:água (50:50), acetonitrila e metanol:água (50:50)).

Para definição do método a ser validado buscando maior seletividade e resolução, também se analisou a variação da tempe- 
ratura do forno $\left(25\right.$ a $\left.80{ }^{\circ} \mathrm{C}\right)$, diferentes proporções de fase móvel (50:50 a 80:20) e diferentes composições de fase móvel (acetonitrila:água e metanol:água).

A performance do sistema cromatográfico foi avaliada a partir dos seguintes parâmetros: fator de capacidade, número de pratos teóricos e fator de cauda, observando-se os cromatogramas obtidos.

\section{Validação do método analítico}

Todo método desenvolvido e não descrito em farmacopéia ou formulários oficiais deve ser validado, sendo avaliados os seguintes parâmetros: especificidade, linearidade, intervalo, precisão, limite de detecção, limite de quantificação, exatidão e robustez ${ }^{11-15}$.

\section{Preparação da solução-amostra}

As amostras foram pesadas analiticamente, diluídas em balão volumétrico com acetonitrila e sonicadas por $15 \mathrm{~min}$. Foi realizada a aferição do volume, filtração em papel de filtro faixa preta marca Schleicher \& Scheuell 589’ ${ }^{\circledR}$. Em seguida, foi amostrada do filtrado uma alíquota volumétrica e diluída com fase móvel acetonitrila:água (50:50) até obtenção da concentração de $100 \mathrm{mg} \mathrm{L}^{-1}$. As amostras foram preparadas em quintuplicatas.

\section{Preparação da curva controle}

Partindo da solução estoque de benznidazol em acetonitrila (1 $\mathrm{mg} \mathrm{mL}^{-1}$ ), foram realizadas diluições em fase móvel (acetonitrila:água (50:50) para obtenção das seguintes concentrações: 40, 100 e $160 \mathrm{mg} \mathrm{L}^{-1}$. A curva controle foi preparada diariamente e utilizada para os cálculos das concentrações.

\section{Equipamentos e condições cromatográficas}

Foi utilizado o cromatógrafo líquido de alta eficiência Shimadzu ${ }^{\circledR}$ composto pelos seguintes módulos: bombas LC -10 ADVP, autoinjetor SIL - 10 ADVP, detector SPD - 10 AVP, controlador SCL - ADVP, forno CTO - 10 ASVP e degaseificador DGU - 14 A.

Utilizou-se balança analítica Sartorius ${ }^{\circledR}$ CP 225 D para realização das pesagens e a lavadora ultrassônica Unique ${ }^{\circledR}$ para a sonicação. As condições cromatográficas definidas a partir do desenvolvimento do método foram: coluna C18, Lichrospher $^{\circledR},(12,5 \mathrm{~cm}$ x 4,0 mm di; partícula $5 \mu \mathrm{m}$ ), fase móvel acetonitrila:água (50:50), fluxo de $1 \mathrm{~mL} \mathrm{~min}^{-1}$, detector UV $316 \mathrm{~nm}$, temperatura do forno de $25^{\circ} \mathrm{C}$ e volume de injeção de $20 \mu \mathrm{L}$. Como pré-requisito para validação de métodos analíticos, os equipamentos e instrumentos foram qualificados e certificados.

\section{Parâmetros avaliados}

Para a realização da robustez foram avaliadas a proporção da fase móvel (acetonitrila:água), variação de fluxo $(0,99$ a 1,01 mL $\min ^{-1}$ ) e tipo de agitação (sonicação e agitador magnético Quimis ${ }^{\circledR}$ ).

A preparação da curva de linearidade foi realizada a partir de três curvas autênticas, nas concentrações 40, 60, 100, 120 e 160 $\mathrm{mg} \mathrm{L}^{-1}$ de benznidazol. Os resultados foram tratados estatisticamente pelo método dos mínimos quadrados, obtendo-se a regressão linear. A faixa de variação testada correspondeu entre 40 a 160\% da concentração em teste, que foi o intervalo entre os limites de quantificação superior e inferior do método.

A especificidade do método foi testada analisando-se comprimidos placebo, contendo amido de milho, lactose malha 200, estearato de magnésio e talco.

A precisão foi avaliada em dois níveis: precisão intra-corrida (repetitividade) e precisão inter-corridas (precisão intermediária). Para a repetitividade, foram preparadas réplicas de 6 determinações a $100 \%$ da concentração teste. Para a precisão intermediária, foram testadas réplicas da concentração teste em dias diferentes e com analistas diferentes.

Os limites de detecção e de quantificação (LD e LQ, respectivamente) foram calculados pela divisão do desvio padrão dos coeficientes lineares das três curvas de calibração do ensaio de linearidade pela média dos coeficientes angulares destas respectivas curvas, multiplicados por 3,0 e 10,0, respectivamente.

A exatidão foi definida preparando-se as análises, considerando que o método é exato quando se analisa a proximidade dos resultados obtidos em relação ao valor verdadeiro, com a utilização do padrão de trabalho como referência. Comprimidos de benznidazol foram manipulados para obtenção das concentrações a 50, 100 e $150 \%$. Em seguida, os resultados foram tratados utilizando-se o teste $\mathrm{t}$ Student, realizando-se uma comparação entre uma média e um valor padrão, com desvio padrão conhecido.

\section{RESULTADOS E DISCUSSÃO}

\section{Desenvolvimento do método analítico}

Após avaliação dos resultados preliminares, onde se considerou a resolução do pico e o tempo de retenção, a fase móvel selecionada seria composta por acetonitrila:água na proporção de 50:50, com fase estacionária, coluna C18, Lichrospher, $12,5 \mathrm{~cm}$ x 4,0 mm di; partícula $5 \mu \mathrm{m}$, justificando uma melhor resolução devido ter a sílica modificada com o grupamento octadecil, visto que o composto hidrofóbico se apresenta com tendência a ficar mais retido. Além disso, o menor comprimento e tamanho de partícula são resultados do desenvolvimento tecnológico das colunas que visam o melhor desempenho cromatográfico ${ }^{20}$.

As amostras foram preparadas com a primeira diluição em acetonitrila, o que se justifica pela hidrofobicidade do ativo e a segunda diluição em fase móvel, opção testada visando a redução de custo para a análise. O cromatograma obtido registrou o pico desta análise simétrico, que se apresenta com tempo de retenção curto, excelente para rotina industrial, agilizando a liberação do produto, principalmente durante o processo de fabricação, como demonstrado na Figura 1.

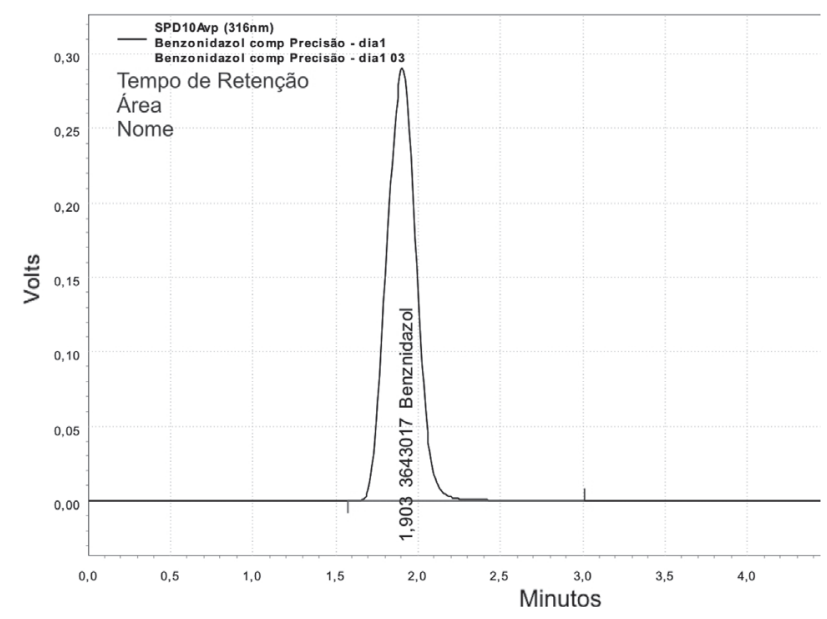

Figura 1. Cromatograma do fármaco no método analítico desenvolvido parâmetro precisão

Após testar diferentes temperaturas para o forno $\left(25\right.$ a $\left.80{ }^{\circ} \mathrm{C}\right)$ foi verificado que, para este método, a temperatura não influenciou na resolução do pico. O fluxo e o volume de injeção foram fixados durante o estudo, considerando-se que se pratica com fre-

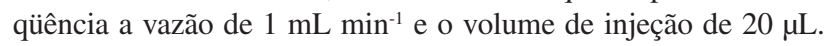


Obteve-se como resultado o número de pratos teóricos de 2659,93 , fator de cauda de 1,22 e fator de capacidade 4,10 conforme Tabela 1.

Tabela 1. Resultados da conformidade do sistema

\begin{tabular}{lc}
\hline Parâmetros & Resultados \\
\hline Pratos teóricos & 2659,93 \\
Fator de cauda $<2$ & 1,22 \\
Fator de capacidade & 4,10 \\
\hline
\end{tabular}

\section{Validação do método analítico}

Os resultados obtidos pelas variações deliberadas aplicadas ao método desenvolvido demonstraram que o mesmo se apresentou robusto para variações de proporções de fase móvel e diferentes tipos de agitação. Porém para as variações de fluxo, no intervalo proposto $(0,99$ a $1,01 \mathrm{~mL})$, verificou-se que há diferença estatiscamente significativa para o intervalo de $95 \%$. Os resultados estão descritos nas Tabelas 2, 3 e 4 . Sendo assim, orienta-se para a utilização deste método o uso do fluxo de $1,0 \mathrm{~mL} / \mathrm{min}$.

Por análise de variância one-way, o valor de $F$ calculado para a variação da proporção da fase móvel foi de 1,040 , sendo inferior ao $F$ tabelado $(3,885)$, já para o fluxo foi de 5,208 , sendo superior ao valor tabelado. Para a variação do tipo de agitação, utilizou-se o tratamento por teste $\mathrm{t}$ Student, obtendo-se como valor de $t$ calculado $(2,287)$ sendo inferior ao $t$ tabelado $(2,306)$, comprovando que não houve diferenças estatisticamente significativas, com $95 \%$ de confiança.

$\mathrm{O}$ método também se mostrou linear, para a faixa de variação entre 40 a $160 \%$, conforme a equação da reta, onde $y=39025,5 x+$ 57455,86 , sendo que $99,85 \%$ dos valores são explicados pela linearidade e que $0,25 \%$ são resíduos; no entanto, seguindo a análise de variância, tem-se que o $\mathrm{R}^{2}$ máximo explicável é de 0,99969. Houve uma regressão estatisticamente significativa e sem falta de ajuste, proporcionando uma curva linear, apresentada na Tabela 5. Ainda como resultado da avaliação da linearidade do método, calculou-se o LQ 1,4960 $\mathrm{mg} \mathrm{L}^{-1}$ e o LD 0,9874 $\mathrm{mg} \mathrm{L}^{-1}$.

Para o ensaio da precisão, na repetitividade de seis réplicas autênticas na concentração de $100 \%$, encontrou-se como resultado a média de $101,91 \mathrm{mg} \mathrm{L}^{-1}$ e desvio padrão relativo de $0,46 \%$, atendendo a resolução vigente. Outro ensaio de precisão, a intermediária, foi testada entre dias e analistas diferentes, tendo como resultado do tratamento estatístico por $\mathrm{t}$ Student que o método foi preciso entre dias e analistas com $95 \%$ de confiança, conforme resultados apresentados nas Tabelas 6 e 7.

Tabela 2. Robustez com relação à proporção da fase

\begin{tabular}{lcccccc}
\hline $\begin{array}{l}\text { Proporção de água ultra pura : } \\
\text { acetonitrila }\end{array}$ & 1 & 2 & 3 & 4 & \multicolumn{3}{c}{ Média } & CV\% \\
\hline $48: 52$ & 105,01 & 104,78 & 104,03 & 103,34 & 105,85 & 104,60 \\
$50: 50$ & 104,52 & 103,27 & 103,28 & 104,31 & 102,57 & 0,92 \\
$52: 48$ & 106,25 & 104,99 & 104,17 & 103,45 & 106,23 & 103,59 \\
\hline
\end{tabular}

Tabela 3. Robustez com relação à variação do fluxo

\begin{tabular}{|c|c|c|c|c|c|c|c|}
\hline \multirow[t]{2}{*}{ Fluxo $\left(\mathrm{mL} \mathrm{min}{ }^{-1}\right)$} & \multicolumn{5}{|c|}{ Concentração $\left(\mathrm{mg} \mathrm{L}^{-1}\right)$} & \multirow[t]{2}{*}{ Média } & \multirow[t]{2}{*}{$\mathrm{CV} \%$} \\
\hline & 1 & 2 & 3 & 4 & 5 & & \\
\hline 0,99 & 103,85 & 102,79 & 101,15 & 102,24 & 102,54 & 102,51 & 0,95 \\
\hline 1,00 & 101,33 & 100,65 & 99,22 & 99,93 & 100,19 & 100,26 & 0,79 \\
\hline 1,01 & 103,69 & 102,72 & 100,52 & 101,13 & 101,03 & 101,82 & 1,31 \\
\hline
\end{tabular}

Tabela 4. Robustez com relação à variação do tipo de agitação

\begin{tabular}{lcccccc}
\hline Tipo de agitação & \multicolumn{3}{c}{ Concentração $\left(\mathrm{mg} \mathrm{L}^{-1}\right)$} & & Média & CV\% \\
& 1 & 2 & 3 & 4 & 5 & 101,43 \\
Sonicação & 102,14 & 100,66 & 101,62 & 101,17 & 101,54 & 0,54 \\
Agitação magnética & 100,14 & 99,90 & 100,88 & 101,17 & 101,01 & 100,62 \\
\hline
\end{tabular}

Tabela 5. Resultados do tratamento estatístico por análise de variância para a regressão linear

\begin{tabular}{|c|c|c|c|c|c|}
\hline Fonte & SQ & GL & MQ & $\mathrm{F}$ & F-crítico \\
\hline Modelo & $4,1669 \mathrm{E}+13$ & 1 & $4,1669 \mathrm{E}+13$ & 8655,87 & 4,6672 \\
\hline Residual & 62581467768 & 13 & 4813959059 & Regressão estatisticamente significativa & \\
\hline Falta de ajuste & 13037095374 & 3 & 4345698458 & 0,87713 & 3,7083 \\
\hline Erro puro & 49544372395 & 10 & 4954437239 & Não há falta de ajuste, proporcionando uma curva linear & \\
\hline Total & $4,17316 \mathrm{E}+13$ & 14 & $2,98083 \mathrm{E}+12$ & & \\
\hline
\end{tabular}

Tabela 6. Resultados da precisão intermediária $-1^{\circ}$ dia

\begin{tabular}{lccccccc}
\hline Analistas & \multicolumn{4}{c}{ Concentração $\left(\mathrm{mg} \mathrm{L}^{-1}\right)$} & \multicolumn{2}{c}{ Média } & \\
& 1 & 2 & 3 & 4 & 5 & \\
\hline I & 101,51 & 101,57 & 101,74 & 101,94 & 101,90 & 101,73 \\
II & 102,48 & 102,36 & 102,04 & 101,36 & 101,36 & 0,19 \\
\hline
\end{tabular}


Tabela 7. Resultados da precisão intermediária $-2^{\circ}$ dia

\begin{tabular}{|c|c|c|c|c|c|c|c|}
\hline \multirow[t]{2}{*}{ Analistas } & \multicolumn{5}{|c|}{ Concentração $\left(\mathrm{mg} \mathrm{L}^{-1}\right)$} & \multirow[t]{2}{*}{ Média } & \multirow[t]{2}{*}{$\mathrm{CV}(\%)$} \\
\hline & 1 & 2 & 3 & 4 & 5 & & \\
\hline I & 102,04 & 102,11 & 102,55 & 101,10 & 101,93 & 101,95 & 0,52 \\
\hline II & 99,44 & 100,53 & 101,95 & 102,57 & 101,08 & 101,11 & 1,21 \\
\hline
\end{tabular}

Em seguida, como resultado do teste para comprovação da especificidade tem-se um cromatograma para o placebo sem detecção no tempo de retenção correspondente ao pico cromatográfico do benznidazol (Figura 2).

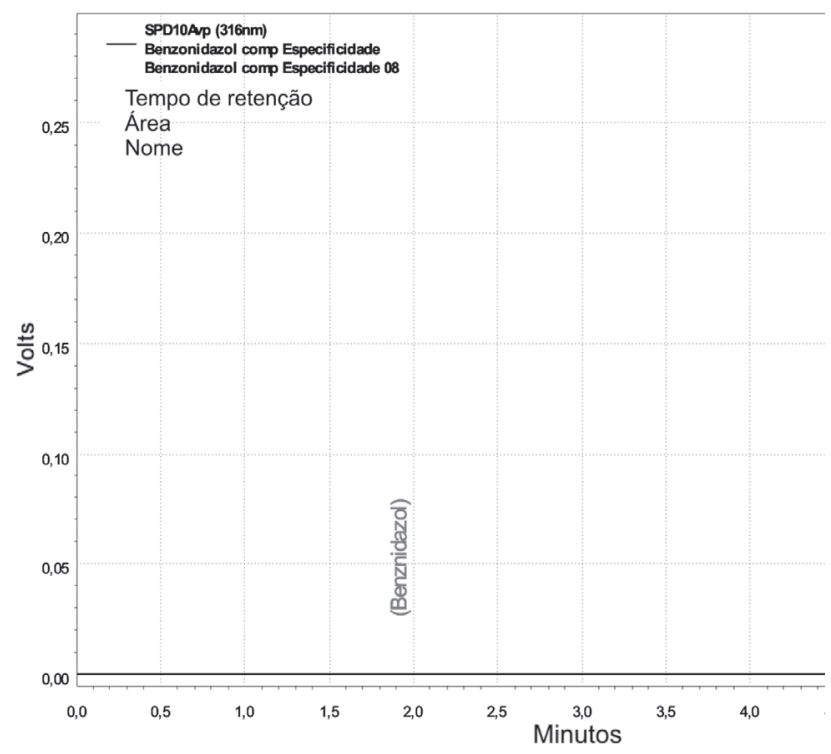

Figura 2. Cromatograma de análise do placebo - parâmetro especificidade

Por fim, o método se mostrou além de específico e exato, visto que, após tratamento estatístico por teste t Student entre comparação de médias, obteve-se para as concentrações 50, 100 e $150 \%$ que os $t$ calculados $(0,6846 ; 2,27$ e 1,566 , respectivamente) foram inferiores ao $t$ tabelado $(2,776)$, com $95 \%$ de confiança.

\section{CONCLUSÃO}

A partir do trabalho realizado tem-se que, pelos resultados apresentados, o método desenvolvido apresenta-se validado conforme a RE no 899 da ANVISA, estando disponível como método específico, preciso, exato e robusto para acompanhamento do estudo de estabilidade nos moldes acelerado e de longa duração dos compri- midos de benznidazol $100 \mathrm{mg}$, desenvolvidos pelo LAFEPE $^{\circledR}$ em parceria com a Roche ${ }^{\circledR}$. Este medicamento é hoje considerado a única alternativa mundial para o tratamento de pacientes crônicos com doença de Chagas.

\section{AGRADECIMENTOS}

Ao Laboratório de Tecnologia dos Medicamentos, da Universidade Federal de Pernambuco e ao Laboratório Farmacêutico do Estado de Pernambuco, LAFEPE.

\section{REFERÊNCIAS}

1. World Health Organization (WHO); WHO Seventeenth Programme Report, Chagas' disease, TDR: Geneve, 2005.

2. Hoare, C. A.; Wallace, F. G.; Nature 1966, 244, 69.

3. http://msf.org.br/informativos/desequilibrio.pdf, acessada em Maio 2006.

4. Malta, J.; Doença de Chagas, Servier: São Paulo, 1996.

5. Brooks, M. A.; Da'rconte, L.; Silva, J. A. F.; J. Pharm. Sci. 1976, 65, 112.

6. Papas, A. N.; Delaney, M. F.; Anal. Lett. 1982, 15, 739.

7. Abu Zuhri, A. Z.; Al-Khalil, S. I.; Suleiman, M. S.; Anal. Lett. 1986, 19, 453.

8. Barbeira, P. J. S.; Silva, G. M.; Beatriz, M. L. P. M.; Stradiotto, N. R.; $J$. Pharm. Biomed. Anal. 1999, 20, 723.

9. Nothenberg, M. S.; Rev. Farm. Bioquim. 1983, 19, 69.

10. Walton, M. I.; Workman, P.; J. Chromatogr. 1986, 375, 190.

11. International Conference on Harmonisation (ICH); Validation of Analytical Procedures: Definitions and Terminology, Q2A (CPMP/ICH/381/95), 1995.

12. International Conference on Harmonisation (ICH); Validation of Analytical Procedures: Methodology, Q2B (CPMP/ICH/281/95), 1995.

13. Agência Nacional de Vigilância Sanitária (ANVISA); Resolução RE $n^{\circ} 899$, de 29 de maio de 2003.

14. de Carvalho e Silva, B.; de Campos, L. M. M.; Nunan, E. A.; Soares, C. D. V.; Silva, G. R.; Ribeiro, J. A. de A.; Pianetti, G. A.; Quim. Nova 2005, $28,50$.

15. Ribani, M.; Bottoli, C. B. G.; Collins, C. H.; Jardim, I. C. S. F.; Melo, L. F. C.; Quim. Nova 2004, 27, 771.

16. Thompson, M.; Ellison, S. L. R.; Wood, R.; Pure Appl. Chem. 2002, 74, 835.

17. Danzer, K.; Otto, M.; Currie, L. A.; Pure Appl. Chem. 1998, 76, 1215.

18. Danzer, K.; Anal. Bioanal. Chem. 2001, 369, 397.

19. Currie, L. A.; Anal. Chim. Acta 1999, 391, 105.

20. Faria, A. M.; Bottoli, C. B. G.; Jardim, I. C. S. F.; Collins, C. H.; Quim. Nova 2006, 29, 300. 